\title{
Optimización del consumo eléctrico mediante la heurística cúmulo de partículas
}

\section{Electrical consumption optimization through particle swarm optimization}

\author{
Blanca Nydia Perez-Camacho' ${ }^{1}$ (D), Juan Manuel Gonzalez-Calleros ${ }^{1}$ (D), Gustavo \\ Rodriguez-Gomez ${ }^{2}$ iD \\ ${ }^{1}$ Benemérita Universidad Autónoma de Puebla, Puebla, México. \\ ${ }^{2}$ Instituto Nacional de Astrofísica, Óptica y Electrónica, Puebla, México. \\ nydia.perez@alumno.buap.mx,juan.gonzalez@correo.buap.mx,grodrig@inaoep.mx
}

(Recibido: 28 julio 2021; aceptado: 13 septiembre 2021; Publicado en Internet: 1 diciembre 2021)

\begin{abstract}
Resumen. En el presente trabajo se da una breve explicación de la técnica de optimización por cúmulo de partículas para ser implementada como parte de la búsqueda del estado óptimo de consumo de un conjunto de dispositivos. Los dispositivos de uso doméstico, en conjunto, permiten caracterizar el consumo eléctrico de una casa habitación a través del comportamiento de uso. Cada uno de los dispositivos presenta un comportamiento de consumo. El objetivo de la optimización se refleja en la función objetivo, la cual es definida de acuerdo con el propósito general de implementación. Los datos de consumo de los dispositivos eléctricos son almacenados en vectores de consumo-hora, donde cada una de las posiciones corresponde al consumo generado por un dispositivo en una hora determinada. Cada uno de los vectores es usado por la heurística como un vector de referencia durante la búsqueda para encontrar el vector que cumple con la función objetivo.
\end{abstract}

Palabras clave: Consumo eléctrico, Optimización del consumo, Cúmulo de partículas, Perfil de uso, Perfil de consumo.

Abstract. This paper gives a brief explanation of the particle swarm optimization technique, which is given to be implemented to look for the optimal state of consumption from a set of household appliances. The household appliances allow characterizing the electrical consumption of a dwelling house through use behavior. Every household appliance shows a behavior consumption. The goal optimization objective is seen as the objective function defined according to the general implementation purpose. The consumption data of household appliances are stored in hourly consumption vectors, where everyone's position corresponds to the consumption generated by a household appliance in each hour. The heuristics use each of the vectors as a reference vector during the search to find the vector that fulfills the objective function.

Keywords: Electrical consumption, Optimized consumption, Particle swarm optimization, User behavior, Consumption behavior.

Tipo de artículo: Artículo de investigación.

\section{Introducción}

En años recientes ha sido objeto de estudio el desarrollo de sistemas que ayuden de manera controlada la manera en la cual se lleva a cabo el consumo eléctrico en casas-habitación (Blecic et al., 2007; Chen et al., 2017; Huang et al., 2015; Sun et al., 2017; Tan et al., 2012). El objetivo primario del control del consumo eléctrico de una casa-habitación está relacionado con la optimización del consumo eléctrico y se enfoca en reducir el consumo de manera que no se vea alterado el perfil del usuario con respecto al uso de los dispositivos (Muhammad Mohsin et al., 2018; Yao et al., 2016; Zhou et al., 2016).

El consumo eléctrico total de una casa es la suma del consumo hora-watts de cada uno de los dispositivos que conforman la infraestructura de dispositivos domésticos. Cualquier dispositivo eléctrico requiere de estar conectado a una fuente de alimentación, ya sea porque para poder funcionar debe de estar conectado o que para poder operar tenga la suficiente carga.

Para el análisis de los datos de consumo hora-watts de los dispositivos, estos de consumo se agrupan en un vector-hora (Adika \& Wang, 2014; Lotfi et al., 2017; Nadeem et al., 2018) y se construyen un vector 
para cada hora del día. Los vectores de consumo-hora son usados por la técnica metaheurística de optimización de cúmulo de partículas (PSO), la cual busca el consumo óptimo de cada uno de los dispositivos de una hora $(t)$, para compararlo con el vector de datos de consumo óptimo que se ha encontrado usando la función objetivo (minimización del consumo) en el espacio de búsqueda.

La presentación de este artículo se organiza de la siguiente manera. En la sección 2 se justifica la selección del algoritmo bioinspirado PSO como técnica de búsqueda de optimización del consumo eléctrico a través de la literatura que trata acerca de su convergencia y del porqué es uno de los algoritmos más usados. La descripción del algoritmo y de las partes que la componen se dan en la sección 3. En la sección 4 , se indica el valor de cada una de las variables usadas en el algoritmo de PSO. Los resultados de la implementación del algoritmo se muestran en la sección 5, y en la sección 6 se enuncian las conclusiones.

\section{Preliminares}

El problema de la búsqueda del consumo óptimo de un conjunto de dispositivos es del tipo np - completo (Huang et al., 2015). Para darle solución a problemas del tipo $n p$-completo se han implementado técnicas de programación lineal, algoritmos genéticos, teoría de juegos, y algoritmos bioinspirados. Siendo los algoritmos bioinspirados los más usados para problemas donde el espacio de búsqueda es amplio (Barbato et al., 2014; Hao et al., 2017; Javaid, Naseem, et al., 2017; Kakran \& Chanana, 2018; Nadeem et al., 2018; Rasheed et al., 2016; Yao et al., 2016)

PSO es un algoritmo bioinspirado desarrollado por Kennedy y Eberhart en el año de 1995 (Kennedy \& Eberhart, 1995). Este algoritmo ha sido analizado teóricamente, y su convergencia ha sido validada en forma exhaustiva. Lo que convierte a PSO en un algoritmo bioinspirado muy confiable dentro de la gama de este tipo de algoritmos (Clerc \& Kennedy, 2002; Emara \& Abdel Fattah, 2004; Jiang et al., 2007; Kadirkamanathan et al., 2006; Kim \& Shin, 2006; Trelea, 2003; van den Bergh \& Engelbrecht, 2006; Zeng et al., 2010; Zhigang Lian et al., 2008). Por el bajo costo computacional, PSO ha sido usado para reducir los costos en el recibo de la energía eléctrica (Huang et al., 2015; Javaid, Hussain, et al., 2017; Rahim et al., 2016). Por los motivos mencionados en los párrafos anteriores, en esta investigación el algoritmo PSO se seleccionó para realizar la búsqueda del consumo óptimo de un conjunto de dispositivos eléctricos.

\section{Descripción del algoritmo PSO}

El algoritmo de optimización por Cúmulo de Partículas (PSO) es la metáfora del comportamiento social que tienen las aves y los peces. PSO es un algoritmo de optimización de búsqueda estocástica conformada por un cúmulo de partículas, dónde cada partícula busca al mejor candidato de solución. El valor de la solución se determina a partir de la evaluación de ella en la función objetivo. Cada una de las partículas se mueve a través del cúmulo. Este movimiento se da ajustando sus trayectorias para continuar su búsqueda de la mejor solución. La posición de cada una de las partículas $x_{i}$ se determina por la velocidad $v_{i}$, el coeficiente de aceleración, la inercia y el tamaño del cúmulo de partículas.

Las ecuaciones que definen la velocidad (1), y la posición de las partículas (2) son:

$$
\begin{gathered}
v_{i j}(t+1)=w v_{i j}(t)+\varphi_{1 j}(t)\left(y_{i j}(t)-x_{i j}(t)\right)+\varphi_{2 j}(t)\left(\hat{y}_{i j}(t)+x_{i j}(t)\right) \\
x_{i j}(t+1)=x_{i j}(t)+v_{1 j}(t+1)
\end{gathered}
$$

En la Tabla 1, se describen los componentes de las ecuaciones (1) y (2).

Tabla 1. Descripción de los componentes de las ecuaciones de velocidad y posición.

\begin{tabular}{|c|l|}
\hline Variables & \multicolumn{1}{|c|}{ Descripción } \\
\hline $\boldsymbol{I}$ & $1, \ldots, s$ \\
\hline $\boldsymbol{J}$ & $1, \ldots, n$ \\
\hline $\boldsymbol{W}$ & Factor inercia, este reduce o aumenta la velocidad de la partícula. \\
\hline
\end{tabular}




\begin{tabular}{|c|l|}
\hline Variables & \multicolumn{1}{|c|}{ Descripción } \\
\hline $\boldsymbol{\varphi}_{\mathbf{1} j}(\boldsymbol{t})$ & $c_{1} r_{1 j}(t)$ \\
\hline $\boldsymbol{\varphi}_{\mathbf{2}}(\boldsymbol{t})$ & $c_{2} r_{2 j}(t)$ \\
\hline $\boldsymbol{s}$ & Es el número de partículas en el enjambre. \\
\hline $\boldsymbol{n}$ & Número de parámetros de la función que va a ser optimizada. \\
\hline $\boldsymbol{c}_{\mathbf{1}}, \boldsymbol{c}_{\mathbf{2}}$ & $\begin{array}{l}\text { Coeficientes de aceleración } 0<c_{1}, c_{2}<1 \text {. Donde } c_{1} \text { es el peso cognitivo y } c_{2} \text { es el peso } \\
\text { social. }\end{array}$ \\
\hline $\boldsymbol{r}_{\mathbf{1}}(\boldsymbol{t}), \boldsymbol{r}_{\mathbf{2}}(\boldsymbol{t})$ & $U(0,1)$, números aleatorios uniformemente distribuidos en el intervalo de $(0,1)$. \\
\hline $\boldsymbol{x}_{\boldsymbol{i}}(\boldsymbol{t})$ & Es la posición de la partícula $i$ en el momento $t$. \\
\hline $\boldsymbol{v}_{\boldsymbol{i}}(\boldsymbol{t})$ & Es la velocidad de la partícula $i$ en el momento $t$. \\
\hline $\boldsymbol{y}_{\boldsymbol{i}}(\boldsymbol{t})$ & Es la mejor solución particular encontrada por la partícula $i$ en el momento $t$. \\
\hline$\widehat{\boldsymbol{y}}_{\boldsymbol{i}}(\boldsymbol{t})$ & Es la mejor posición global encontrada por la partícula $i$ en el momento $t$. \\
\hline
\end{tabular}

Para garantizar la convergencia del algoritmo PSO debe de cumplirse que $0<c_{1}, c_{2}<1$, y $r_{1}$, $r_{2}$ son números aleatorios uniformemente distribuidos en el intervalo de (0,1) (Jiang et al., 2007; Kim \& Shin, 2006; Trelea, 2003; van den Bergh \& Engelbrecht, 2006). La ecuación (1), trata sobre la velocidad de la partícula. Dicha velocidad es determinada por los componentes que se mencionan en la Tabla 2.

Tabla 2. Componentes de la ecuación de velocidad.

\begin{tabular}{|c|c|}
\hline Componente & Función \\
\hline$v_{i}(t)$ & Sirve para prevenir la oscilación en la búsqueda de la dirección. \\
\hline $\begin{array}{l}\varphi_{1}(t)\left(y_{i}(t)\right. \\
\left.-x_{i}(t)\right)\end{array}$ & $\begin{array}{l}\text { Componente cognitivo. El cual representa la distancia de la partícula a la mejor solución } \\
y_{i}(t) \text { encontrada por ella. El componente cognitivo le sirve a cada partícula para regresar a } \\
\text { donde ha encontrado la mejor solución. }\end{array}$ \\
\hline $\begin{array}{l}\varphi_{2}(t)\left(\widehat{y}_{i}(t)\right. \\
\left.-x_{i}(t)\right)\end{array}$ & $\begin{array}{l}\text { Componente social. Este representa la distancia entre la partícula y la mejor posición global. El } \\
\text { componente social representa la dependencia de cada partícula para lograr el éxito de las otras } \\
\text { partículas. }\end{array}$ \\
\hline$\widehat{y}_{i}(t)$ & Representa la mejor solución global encontrada por la partícula $i$. \\
\hline
\end{tabular}

A continuación es presentado el pseudo código (Pseudo Código 1). El diagrama de flujo de PSO se muestra en la Figura 1.

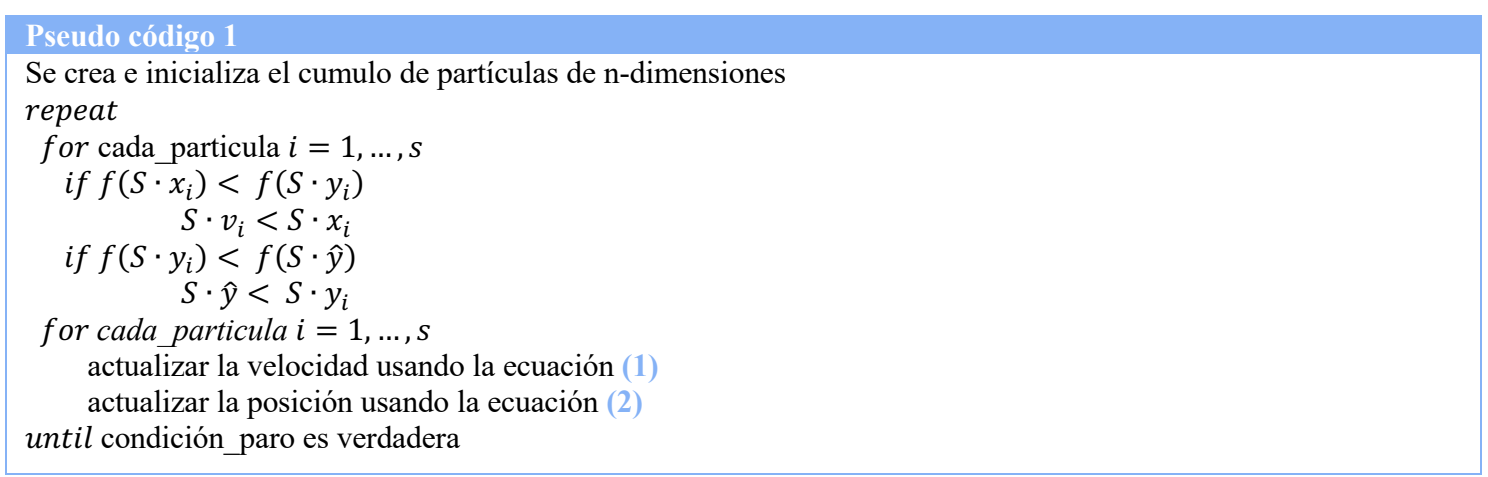




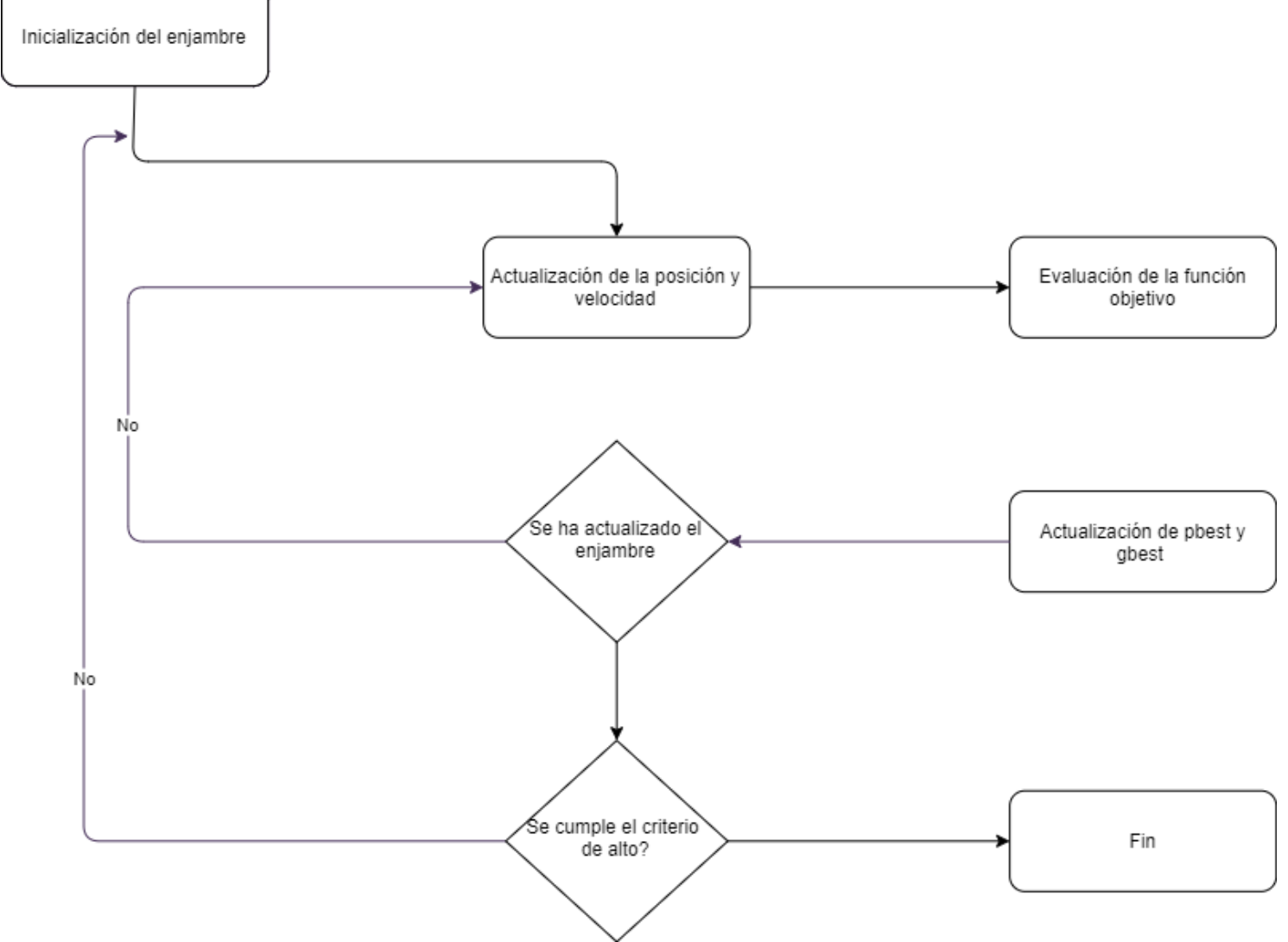

Figura 1. Diagrama de flujo de PSO.

\section{PSO aplicado a la búsqueda del consumo óptimo}

Para el caso particular de aplicación del algoritmo PSO en la búsqueda del estado óptimo de un conjunto de dispositivos, dónde el espacio de búsqueda se conforma por los vectores de consumo de diez dispositivos, y un enjambre de 1000 partículas; la función objetivo de la implementación de PSO queda definida como el mínimo de la suma de los consumos del conjunto de los (caso particular) diez dispositivos.

Para el experimento, se definen los parámetros de inercia en 0.8 , el peso cognitivo y social se ponen en uno. Los criterios de paro de la ejecución del programa son, ya sea, de 50 ciclos o cuando el valor mínimo ya se ha repetido 5 veces. El código del algoritmo se implementó en el lenguaje Python usando el editor Jupyter en una máquina Dell Inspiron 155000 series con un procesador Intel Core i7, memoria RAM de 8.0 Gb, y sistema operativo Windows 10 . La correspondencia del problema particular a resolver con el algoritmo PSO son:

Cada uno de los elementos del espacio de búsqueda es representado mediante un vector de diez parámetros $[d 1, \ldots d 10]$, dónde cada parámetro corresponde al consumo de un dispositivo en el momento $m$. Cada vector del espacio de búsqueda se crea colocando en cada parámetro un valor aleatorio obtenido del rango del consumo mínimo (cMin) y el consumo máximo (cMax) correspondientes al dispositivo.

El vector queda de la siguiente manera:

[ventiladorconsumo, aireacondicionadoconsumo, estufaconsumo, fococonsumo, calefactorconsumo, lavatrastesconsumo, microondasconsumo, secadoraconsumo, lavadoraconsumo, refrigeradorconsumo]

Se generan 1000 partículas. El mejor valor encontrado por la partícula que cumple con la función objetivo es almacenado en la variable bpart. Cuando la partícula ha terminado de realizar la búsqueda se compara el valor almacenado en su variable bpart con respecto al valor almacenado en la variable $b g l o b a l$. 
En bglobal se almacena el mejor de los valores encontrados por todas las partículas que cumplen con la función objetivo. Esta variable es inicializada con el valor de la bpart de la primera partícula, posteriormente el valor de la bglobal es comparado con el valor bpart de cada una de las partículas, y si alguna de ellas es mejor, de acuerdo con la función objetivo, entonces se le es asignado ese valor.

Función objetivo: para este caso de estudio el objetivo es el de minimizar el consumo eléctrico. La función objetivo se define como min $\sum_{a_{i} \in A} \gamma_{a_{i}}^{\tau}$. Dónde $A$ es el conjunto de dispositivos, $a_{i}$ es un elemento del conjunto $A, \Gamma$ es una hora del día y se encuentra en el rango $[0,23], \gamma_{a_{i}}^{\tau}$ es el consumo por hora del elemento $a_{i}$ en la hora $\Gamma$.

En la función objetivo es evaluado cada uno de los elementos del espacio de búsqueda. Si alguno de ellos cumple con el criterio de ser menor que el valor actual almacenado en bglobal entonces son actualizados por los nuevos valores encontrados tanto la posición como el valor.

\section{Resultados}

PSO opera usando dos bases de datos: una de consumo y otra de estado. Ambas están conformadas por 365 registros (representan los 365 días del año) de 24 columnas. Cada columna corresponde a una hora del día. Cada uno de los registros es usado por la técnica PSO como parámetros de estado y consumo para cada uno de los dispositivos. Cada uno de estos registros de consumo sirve como vector de comparación para el vector de consumo encontrado por PSO (ver Figura 2).

Se hicieron 100 repeticiones del experimento (100 propuestas de consumo para cada uno de los perfiles de consumo hora - día $\times 365$ registros). Se efectuó un análisis estadístico con los datos obtenidos de cada uno de los experimentos obteniendo el promedio de los cien experimentos, esto para cada uno de los diez dispositivos, lo cual representa el consumo promedio para cada uno de estos. Es calculada, también, la desviación estándar de acuerdo con el dispositivo relación experimento. Luego es calculado el promedio de las desviaciones de los 100 experimentos. Los resultados obtenidos se muestran en la Tabla 3. Como parte de los resultados se observa que los siguientes cuatro dispositivos: estufa, foco, lavadora y secadora, presentan un mismo consumo sin importar la función que estén realizando. Así, los cuatros dispositivos mencionados presentan como promedio de consumo un consumo normal, y una desviación estándar de cero pues no hay variación en el consumo.

Tabla 3. Análisis de los datos de consumo propuestos por PSO

\begin{tabular}{|l|l|l|}
\hline \multicolumn{1}{|c|}{ Dispositivo } & \multicolumn{1}{c|}{ Desviación estándar } \\
\hline Aire Acondicionado & 1496.341 & $9.0 \mathrm{e}-5$ \\
\hline Calefactor & 8.0800005 & $5.760072 \mathrm{e}-02$ \\
\hline${ }^{*}$ Estufa & 1425.0 & 0 \\
\hline${ }^{*}$ Foco & 10.5 & 0 \\
\hline${ }^{*}$ Lavadora & 895.5 & 0 \\
\hline Lava trastes & 803.1705 & $2.25 \mathrm{e}-05$ \\
\hline Microondas & 1082.43 & $9 \mathrm{e}-11$ \\
\hline Refrigerador & 2861.7000000000003 & $4.54747350886461 \mathrm{e}-13$ \\
\hline${ }^{*}$ Secadora & 980.625 & 0 \\
\hline Ventilador & 88.81999999999998 & $1.4210854715202004 \mathrm{e}-14$ \\
\hline
\end{tabular}


Consumo de los dispositivos

\begin{tabular}{|c|c|c|c|c|c|c|c|c|c|c|}
\hline Hora & Calefactor & Aire & Ventilador & Secadora & Lavatrastes & Estufa & Microondas & Lavadora & Refrigerador & Foco \\
\hline 0.0 & 0.0 & 0.0 & 0.0 & 0.0 & 836.783881 & 1425.0 & 1211.428365 & 0.0 & 0.0 & 0.0 \\
\hline
\end{tabular}

Reducción: 162.61024614127928

\% Reducción: 4.681842866320531

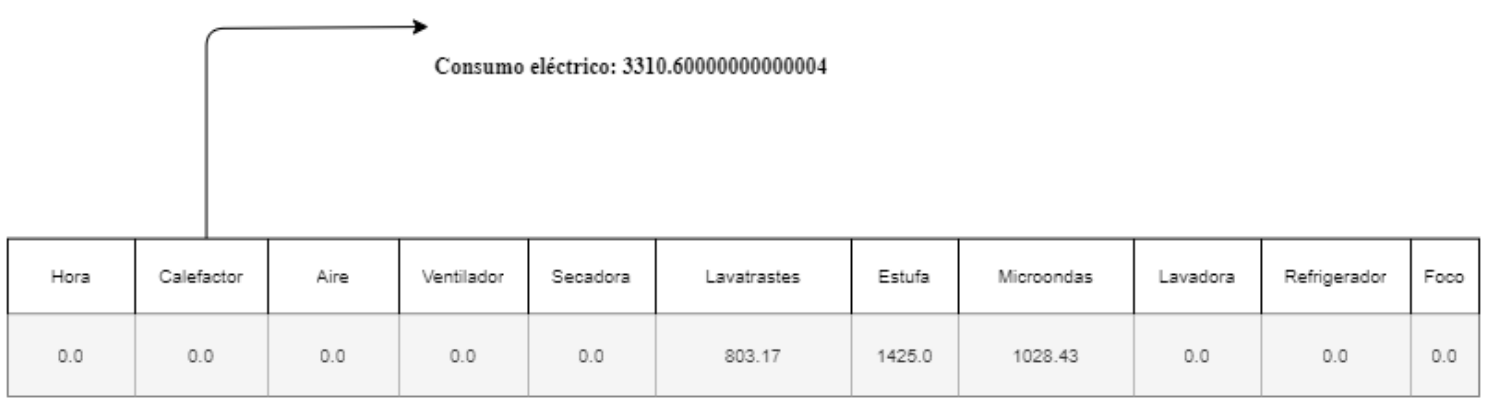

Consumo propuesto por PSO

Figura 2. Consumo contra consumo propuesto por PSO

\section{Conclusiones}

En este artículo, se presentó la implementación de la técnica de cúmulo de partículas como método de búsqueda del estado óptimo de consumo de los dispositivos que es encontrado a partir del procesamiento del consumo eléctrico de un conjunto de diez dispositivos de uso doméstico.

La búsqueda del consumo óptimo se obtiene a partir de la definición de la función objetivo, pues esta sirve para determinar el objetivo de implementación de la técnica de optimización.

La implementación de PSO permite proponer el consumo que cada uno de los dispositivos debería de consumir con el objetivo de bajar el consumo total sin necesidad de apagar alguno de los dispositivos que se encuentran en estado activo.

\section{Declaración de conflicto de intereses}

Los autores declaran no tener conflicto de intereses con respecto a la investigación, autoría o publicación de este artículo.

\section{Financiación}

Los autores no recibieron apoyo financiero para la investigación, autoría y/o publicación de este artículo. 


\title{
Agradecimientos
}

Al programa de LKE de la Facultad de Ciencias de la Computación de la BUAP por permitir el uso de las instalaciones, así como también del apoyo de los catedráticos que conforman a su cuerpo académico por su guía y dedicación.

\section{ORCID iD}

\author{
Blanca Nydia Perez-Camacho (iD https://orcid.org/0000-0002-2334-8806 \\ Juan Manuel González-Calleros (iD https://orcid.org/0000-0002-9661-3615 \\ Gustavo Rodriguez-Gomez (iD) https://orcid.org/0000-0002-4925-8892
}

\section{Referencias}

Adika, C. O., \& Wang, L. (2014). Autonomous Appliance Scheduling for Household Energy Management. IEEE Transactions on Smart Grid, 5(2). https://doi.org/10.1109/TSG.2013.2271427

Barbato, A., Capone, A., Carello, G., Delfanti, M., Falabretti, D., \& Merlo, M. (2014). A framework for home energy management and its experimental validation. Energy Efficiency, 7(6). https://doi.org/10.1007/s12053-014-92693

Blecic, I., Cecchini, A., \& Trunfio, G. A. (2007). A decision support tool coupling a causal model and a multi-objective genetic algorithm. Applied Intelligence, 26(2). https://doi.org/10.1007/s10489-006-0009-Z

Chen, S., Liu, T., Gao, F., Ji, J., Xu, Z., Qian, B., Wu, H., \& Guan, X. (2017). Butler, Not Servant: A Human-Centric Smart Home Energy Management System. IEEE Communications Magazine, 55(2). https://doi.org/10.1109/MCOM.2017.1600699CM

Clerc, M., \& Kennedy, J. (2002). The particle swarm - explosion, stability, and convergence in a multidimensional complex space. IEEE Transactions on Evolutionary Computation, 6(1). https://doi.org/10.1109/4235.985692.

Emara, H. M., \& Abdel Fattah, H. A. (2004). Continuous swarm optimization technique with stability analysis. Proceedings of the 2004 American Control Conference, 2811-2817. https://doi.org/10.23919/ACC.2004.1383892

Hao, Y., Wang, W., \& Qi, Y. (2017, October). Optimal home energy management with PV system in time of use tariff environment. 2017 Chinese Automation Congress (CAC). https://doi.org/10.1109/CAC.2017.8243232

Huang, Y., Tian, H., \& Wang, L. (2015). Demand response for home energy management system. International Journal of Electrical Power \& Energy Systems, 73. https://doi.org/10.1016/j.ijepes.2015.05.032

Javaid, N., Hussain, S., Ullah, I., Noor, M., Abdul, W., Almogren, A., \& Alamri, A. (2017). Demand Side Management in Nearly Zero Energy Buildings Using Heuristic Optimizations. Energies, 10(8). https://doi.org/10.3390/en10081131

Javaid, N., Naseem, M., Rasheed, M. B., Mahmood, D., Khan, S. A., Alrajeh, N., \& Iqbal, Z. (2017). A new heuristically optimized Home Energy Management controller for smart grid. Sustainable Cities and Society, 34. https://doi.org/10.1016/i.scs.2017.06.009

Jiang, M., Luo, Y. P., \& Yang, S. Y. (2007). Stochastic convergence analysis and parameter selection of the standard particle swarm optimization algorithm. Information Processing Letters, 102(1). https://doi.org/10.1016/j.ipl.2006.10.005

Kadirkamanathan, V., Selvarajah, K., \& Fleming, P. J. (2006). Stability analysis of the particle dynamics in particle swarm optimizer. IEEE Transactions on Evolutionary Computation, 10(3), 245-255. https://doi.org/10.1109/TEVC.2005.857077

Kakran, S., \& Chanana, S. (2018). Energy Scheduling of Smart Appliances at Home under the Effect of Dynamic Pricing Schemes and Small Renewable Energy Source. International Journal of Emerging Electric Power Systems, 19(2). https://doi.org/10.1515/ijeeps-2017-0187

Kennedy, J., \& Eberhart, R. (1995). Particle swarm optimization. Proceedings of ICNN'95 - International Conference on Neural Networks. https://doi.org/10.1109/ICNN.1995.488968

Kim, D. H., \& Shin, S. (2006). Self-organization of Decentralized Swarm Agents Based on Modified Particle Swarm Algorithm. Journal of Intelligent and Robotic Systems, 46(2). https://doi.org/10.1007/s 10846-006-9047-3

Lotfi, J., Abdi, F., \& Abbou, M. F. (2017, November). Smart Home Energy System Modeling and Implementation. 2017 European Conference on Electrical Engineering and Computer Science (EECS). https://doi.org/10.1109/EECS.2017.80 
Muhammad Mohsin, S., Javaid, N., Madani, S. A., Abbas, S. K., Akber, S. M. A., \& Khan, Z. A. (2018, May). Appliance Scheduling in Smart Homes with Harmony Search Algorithm for Different Operation Time Intervals. 2018 32nd International Conference on Advanced Information Networking and Applications Workshops (WAINA). https://doi.org/10.1109/WAINA.2018.00063

Nadeem, Z., Javaid, N., Malik, A., \& Iqbal, S. (2018). Scheduling Appliances with GA, TLBO, FA, OSR and Their Hybrids Using Chance Constrained Optimization for Smart Homes. Energies, 11(4). https://doi.org/10.3390/en11040888

Rahim, S., Javaid, N., Ahmad, A., Khan, S. A., Khan, Z. A., Alrajeh, N., \& Qasim, U. (2016). Exploiting heuristic algorithms to efficiently utilize energy management controllers with renewable energy sources. Energy and Buildings, 129. https://doi.org/10.1016/j.enbuild.2016.08.008

Rasheed, M., Javaid, N., Awais, M., Khan, Z., Qasim, U., Alrajeh, N., Iqbal, Z., \& Javaid, Q. (2016). Real Time Information Based Energy Management Using Customer Preferences and Dynamic Pricing in Smart Homes. Energies, 9(7). https://doi.org/10.3390/en9070542

Sun, X., Ji, S., \& Wen, C. (2017, October). An optimized scheduling strategy for smart home users under the limitation of daily electric charge. 2017 Chinese Automation Congress (CAC). https://doi.org/10.1109/CAC.2017.8244020

Tan, X., Shan, B., Hu, Z., \& Wu, S. (2012, June). Study on demand side management decision supporting system. 2012 IEEE International Conference on Computer Science and Automation Engineering. https://doi.org/10.1109/ICSESS.2012.6269417

Trelea, I. C. (2003). The particle swarm optimization algorithm: convergence analysis and parameter selection. Information Processing Letters, 85(6). https://oi.org/10.1016/S0020-0190(02)00447-7

van den Bergh, F., \& Engelbrecht, A. P. (2006). A study of particle swarm optimization particle trajectories. Information Sciences, 176(8). https://doi.org/10.1016/j.ins.2005.02.003

Yao, L., Shen, J.-Y., \& Lim, W. H. (2016, December). Real-Time Energy Management Optimization for Smart Household. 2016 IEEE International Conference on Internet of Things (IThings) and IEEE Green Computing and Communications (GreenCom) and IEEE Cyber, Physical and Social Computing (CPSCom) and IEEE Smart Data (SmartData). https://doi.org/10.1109/iThings-GreenCom-CPSCom-SmartData.2016.31

Zeng, W., Zhang, Y., \& Yan, L. (2010, October). Mechanism of Particle Swarm Optimization and Analysis on Its Convergence. 2010 Third International Symposium on Information Processing. https://doi.org/10.1109/ISIP.2010.46

Zhigang Lian, Fan Zhu, Zailin Guan, \& Xinyu Shao. (2008). The analysis of particle swarm optimization algorithm's convergence. 20087 th World Congress on Intelligent Control and Automation. https://doi.org/10.1109/WCICA.2008.4592994

Zhou, B., Li, W., Chan, K. W., Cao, Y., Kuang, Y., Liu, X., \& Wang, X. (2016). Smart home energy management systems: Concept, configurations, and scheduling strategies. Renewable and Sustainable Energy Reviews, 61. https://doi.org/10.1016/j.rser.2016.03.047 